\title{
Baumann Skin Type in the Korean Female Population
}

\author{
Sung Ku Ahn, Myungsoo Jun, Hana Bak, Byung Duk Park ${ }^{1}$, Seung Phil Hong ${ }^{2}$, Sang-Hoon Lee ${ }^{3}$, \\ Seong-Jin Kim ${ }^{4}$, Hyung Joo Kim ${ }^{5}$, Dong Hoon Song ${ }^{5}$, Pok Kee Min ${ }^{6}$, Ja Woong Goo, Tae-Hyun Kim, \\ Chang-Keun $\mathrm{Oh}^{7}$, Seung Hyun Chun, Sewon Lee, Yeol Oh Sung ${ }^{6}$, In-Bum Sohn, Hyung Jin Ahn, Kun Park ${ }^{8}$ \\ Department of Dermatology, Yonsei University Wonju College of Medicine, Wonju, ${ }^{1}$ Neopharm Co., Ltd., Daejeon, ${ }^{2}$ Department of \\ Dermatology, Dankook University Medical College, Cheonan, ${ }^{3}$ Department of Dermatology, Soonchunhyang University College of \\ Medicine, Seoul, ${ }^{4}$ Department of Dermatology, Chonnam National University Medical School, Gwangju, ${ }^{5}$ Department of Dermatology, \\ Yonsei University College of Medicine, Seoul, ${ }^{6}$ Department of Dermatology, Kyungpook National University School of Medicine, Daegu, \\ ${ }^{7}$ Department of Dermatology, Pusan National University School of Medicine, Busan, ${ }^{8}$ Department of Dermatology, Wonkwang University \\ School of Medicine, Iksan, Korea
}

Background: To meet the need for a subspecialized skin type system, the Baumann skin type (BST) system was proposed. Objective: To evaluate the distribution of BST types and influencing factors among Korean women. Methods: BST questionnaires were administered to 1,000 Korean women. The possible responses were as follows: oily $(\mathrm{O})$ or dry (D), sensitive $(S)$ or resistant $(R)$, pigmented $(P)$ or non-pigmented $(\mathrm{N})$, and wrinkled $(\mathrm{W})$ or tight $(\mathrm{T})$. The correlations of the BST with the subjects' age, location, ultraviolet (UV) ray exposure, drinking and smoking habits, and blood type were assessed. Results: The OSNT, DSNT, DRNT, and OSNW skin types were the most common skin types (55.3\%). The O, S, P, and W types accounted for $46.6 \%, 68.8 \%, 23.2 \%$, and $31.9 \%$, respectively. The proportion of the $\mathrm{O}$ and $\mathrm{S}$ type was the highest in Gyeongsangbuk-do (55.0\%) and Seoul $(77.2 \%)$. The proportion of the $\mathrm{P}$ and $\mathrm{W}$ type was the highest in Gyeongsangbuk-do (33.0\%) and Chungcheong-do $(39.0 \%)$. The $\mathrm{O}$ type decreased in the higher age group, whereas the $\mathrm{P}$ and $\mathrm{W}$ type showed a reversed tendency. In smokers, the proportion of W type was significantly higher than in the non-smokers $(66.3 \%$ vs. $24.1 \%, p<0.05)$.

Received June 30, 2016, Revised December 30, 2016, Accepted for publication February 8, 2017

Corresponding author: Sung Ku Ahn, Department of Dermatology, Wonju Severance Christian Hospital, 20 Ilsan-ro, Wonju 26426, Korea. Tel: 82-33-741-0621, Fax: 82-33-748-2650, E-mail: ahnsk@yonsei.ac.kr

This is an Open Access article distributed under the terms of the Creative Commons Attribution Non-Commercial License (http://creativecommons. org/licenses/by-nc/4.0) which permits unrestricted non-commercial use, distribution, and reproduction in any medium, provided the original work is properly cited.

Copyright (c) The Korean Dermatological Association and The Korean Society for Investigative Dermatology
Conclusion: The 4 most common BST types were OSNT, DSNT, DRNT, and OSNW. In the comparison across the 4 BST parameters according to the age, region, smoking and drinking habits, occupation, blood type, and UV exposure, significant differences were observed. Individualized and customized skin care is required according to the personal skin type. (Ann Dermatol 29(5) 586 596, 2017)

\section{-Keywords-}

4 Parameters, Baumann skin type, Korean women, Surveys and questionnaires

\section{INTRODUCTION}

The skin is a complex organ that protects its host from its environment and concurrently allows for interactions between the host and its environment. With age and exposure to harmful environmental irritants such as ultraviolet (UV) radiation, the skin loses its functional integrity and gradually becomes damaged. With the emerging need for personalized and more tailored skin care, various skin-typing systems have been proposed.

In 1910, Helena Rubinstein suggested a primary classification of skin types as follows: dry, oily, combination, and sensitive. Although these skin type categories are still widely used in the skin care industry, the lack of more sophisticated descriptions of skin conditions such as pigmentation or wrinkles, and the discrepancies between subjective and objective assessments raise the need for the development of further subspecialized skin type categories. The Baumann skin type (BST) system was proposed in 
$2006^{1}$, and it is composed of 4 distinct spectra, namely dry to oily, sensitive to resistant, pigmented to non-pigmented, and wrinkled to tight.

Accordingly, we performed a descriptive study to identify characteristic skin types among Korean women with the purpose of determining the most common skin type and ascertaining the regional and age-group distributions. Based on the results of this study, the most characteristic skin features of Korean women can be identified, allowing for the provision of more discriminate and individualized skin care for diverse skin conditions.

\section{MATERIALS AND METHODS}

\section{Subjects}

This study was approved by the Yonsei University Wonju Severance Christian Hospital Institutional Review Board (IRB no. CR316029). Data were collected from 1,000 healthy Korean female volunteers without skin disease (Table 1). The subjects' basic information was analyzed according to the subjects' age and location and data obtained from Baumann's questionnaire.

Table 1. Basic information of the subjects

\begin{tabular}{lc}
\hline \multicolumn{1}{c}{ Variable } & $\mathrm{n}(\%)$ \\
\hline Region $(\mathrm{n}=1,000)$ & $250(25.0)$ \\
Seoul & $250(25.0)$ \\
Gyeonggi-do/Incheon & $100(10.0)$ \\
Gyeongsangnam-do & $100(10.0)$ \\
Jeolla-do & $100(10.0)$ \\
Gyeongsangbuk-do & $100(10.0)$ \\
Chungcheong-do & $70(7.0)$ \\
Gangwon-do & $30(3.0)$ \\
Jeju-do & \\
Age (yr, $\mathrm{n}=1,000)$ & $255(25.5)$ \\
$20 \sim 29$ & $253(25.3)$ \\
$30 \sim 39$ & $248(24.8)$ \\
$40 \sim 49$ & $244(24.4)$ \\
$50 \sim 59$ & $792(96.9)$ \\
Working environment $(\mathrm{n}=817)$ & $25(3.1)$ \\
Mainly indoor* & \\
Mainly outdoor & \\
Blood type (n=837) & $299(35.7)$ \\
A & $216(25.8)$ \\
B & $229(27.4)$ \\
O & $93(11.1)$ \\
AB &
\end{tabular}

*Indoor occupation includes health-care providers, school personnel, singer, designers, hair designers, or painters. ${ }^{\dagger}$ Outdoor occupation includes construction industry workers, military personnel, sports instructors, transportation industry workers, taxi drivers, and farmers.

\section{Baumann skin type questionnaire}

The BST of each subject was determined by the questionnaire (representative parts of the questionnaire are presented as Appendix 1), which included 4 parts and a total of 63 questions. In each part, the natural moisturizing capacity and sebum production of the skin, skin sensitivity to external irritants, personal or family history of melanin-related skin diseases, and tendency to develop pigmentation after injury or skin reaction to UV ray exposure are evaluated. Each question can be answered with $1 \sim 4$ points according to the relevance, and 2.5 points were assigned for ambiguous cases. After the questionnaire was completed, a combination of the 4 parameters in each part was used to assign one particular skin type to each participant.

\section{Distribution of the 16 Baumann skin types and Baumann's 4 parameters}

The nationalwide distribution and age-specific distribution were analyzed. For more detailed analysis, the distribution of Baumann's 4 parameters (dry [D] or oily [O], sensitive [S] or resistant [R], wrinkled [W] or tight [T], and pigmented [P] or non-pigmented [N]) were compared according to the subjects' age and location.

\section{Miscellaneous factors that may have influenced the skin type distribution}

In the BST questionnaire, information about smoking, drinking, UV exposure, use of sunblocks, and exposure to air pollution can be collected. In addition, information about the subjects' occupation and blood type was collected. The relevance of these factors to skin type distribution was also investigated.

\section{Statistical analyses}

To compare the proportion of the subjects in each group, the chi-square, Fisher exact, and Mantel-Haenszel tests were used. IBM SPSS Statistics ver. 21.0 (IBM Co., Armonk, $\mathrm{NY}$, USA) was used for the statistical analyses. A $p$-value of $<0.05$ was defined as statistically significant.

\section{RESULTS}

\section{Distribution of the 16 Baumann skin types}

\section{1) Nationwide distribution}

The most common skin type among South Korean women was OSNT, followed by DSNT, DRNT, OSNW, ORNT, and DSNW (Fig. 1). The top 4 types accounted for $55.3 \%$ of the subjects. Each of the other skin types comprised 
$<10 \%$ of the study population.

\section{2) Regional distribution}

In Gyeonggi-do/Incheon, Gyeongsangbuk-do, Gyeongsangnam-do, and Jeju-do, OSNT was the most common BST, as in the nationwide analysis. However, in Gangwon-do, Seoul, and Chungcheong-do, DSNT was the most common skin type. In Jeolla-do, DRNT, which ranked third or lower in other regions, was the most common skin type. In most of the regions, the top 3 types comprised $\geq 50 \%$ of the study population (Fig. 2).

\section{3) Age-specific distribution}

Among women in their twenties, thirties, and forties, the most common skin type was OSNT, followed by DSNT and DRNT. The OSNW type held the fourth rank among women in their twenties and thirties. In those in their forties, the OSNW type held the fifth rank after ORNT (Fig. 3). Among women in their fifties, DSNT, which was the second most common skin type in the other age groups, was the most common skin type, followed by OSNT, which was the most common skin type in other age groups. In addition, DSPT, which comprised $\leq 10 \%$ of the women in their twenties and thirties, accounted for $10.4 \%$, holding fourth rank (Fig. 3).

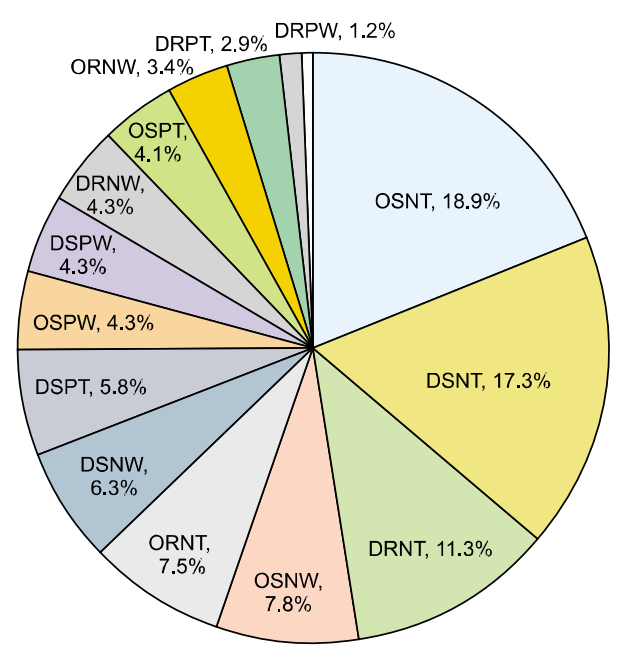

Fig. 1. The nationwide distribution of the Baumann skin type in the Korean female population. The proportion of each skin type is arranged in a clockwise, decreasing manner. Of the 16 Baumann skin types, the top 4 types account for $>50 \%$. O: oily, D: dry, S: sensitive, R: resistant, P: pigmented, N: non-pigmented, W: wrinkled, $\mathrm{T}$ : tight.

\section{Distribution of Baumann's 4 parameters}

\section{1) Nationwide distribution}

In general, $53.4 \%$ of the Korean women were assessed to have dry skin, and $68.8 \%$ had sensitive skin. Only $23.2 \%$ and $31.9 \%$ of the Korean women had pigmented and wrinkled skin, respectively (Fig. 4).

\section{2) Age-specific distribution}

The age-specific distribution of Baumann's 4 parameters is schematized in Appendix 2. In the twenties age group, $52.9 \%$ of the subjects had oily skin. However, the proportion of subjects with oily skin decreased as age increased, reaching $32.8 \%$ in the fifties age group. The differences in the proportion of subjects with oily skin were statistically significant between each age group $(p<0.001)$. Among the subjects in their twenties, $71.4 \%$ had sensitive skin, and the proportion reached its highest in the thirties $(75.1 \%)$. It decreased as the subjects' age increased further, reaching $62.3 \%$ in the fifties age group, with statistically significant differences between each age group $(p=0.011)$. The proportion of subjects with pigmented or wrinkled skin increased as the subjects' age increased, reaching $29.5 \%$ and $36.9 \%$ in the fifties age groups, respectively. For pigmented and wrinkled skin, the differences between each age group were statistically significant ( $p<0.001$ and $p=0.016$, respectively).

\section{3) Regional distribution}

From the perspective of regional differences in skin type, the oily skin type was the most common in Gyeongsangbuk-do and the least common in Jeju-do $(55.0 \%$ and $40.0 \%$, respectively). However, the differences between the groups by location were not statistically significant $(p>0.05)$. The proportion of subjects with sensitive skin type was the highest in Seoul $(77.2 \%)$, and the lowest in Jeolla-do $(50.0 \%)$. The differences between each group by location were statistically significant $(p<0.01)$. The proportion of pigmented skin type was highest in Gyeongsangbuk-do (33.0\%) and lowest in Gangwon-do (18.6\%). The proportion of those with wrinkled skin type was the highest in Chungcheong-do (39.0\%) and the lowest in Seoul $(25.6 \%)$. However, the differences between the proportion of subjects with pigmented or wrinkled skin type were not statistically significant between locations ( $p>$ 0.05). A detailed distribution of Baumann's 4 parameters according to the location of the subjects was schematized in Appendix 3.

The distribution of Baumann's 4 parameters was compared according to the latitude of the location of the 
A
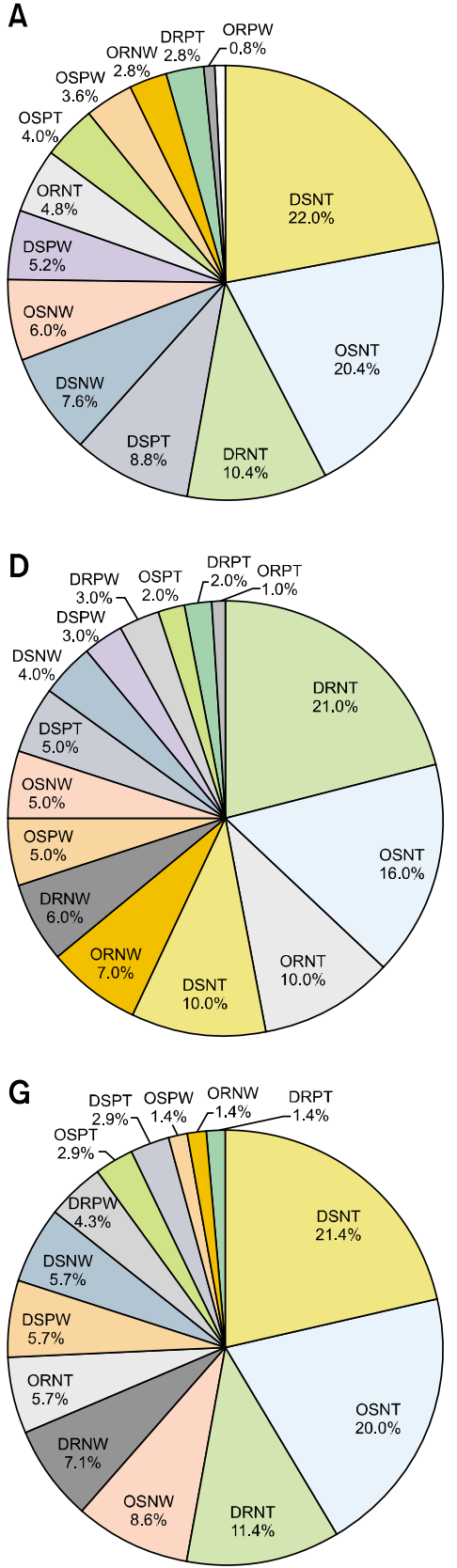

B

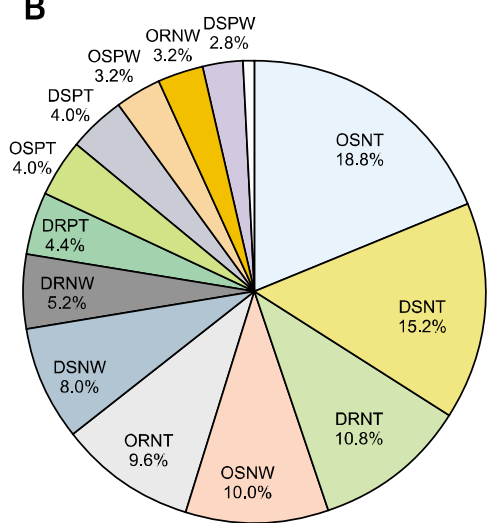

$E$

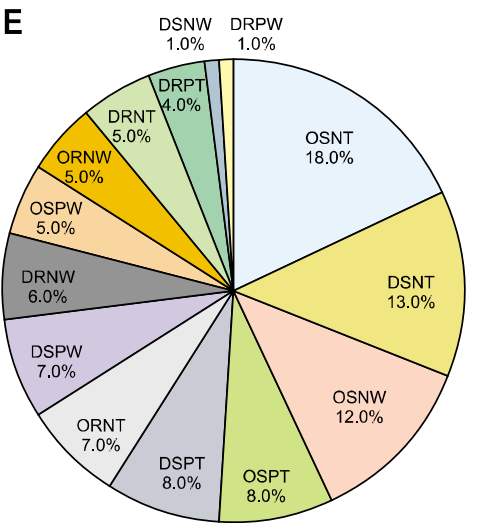

H

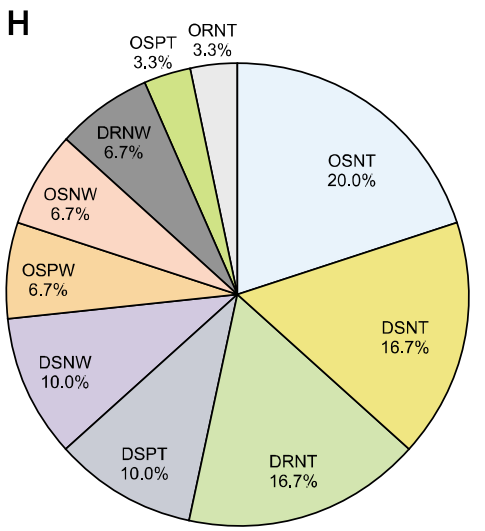

C DRPT DRNW ORPT ORNW

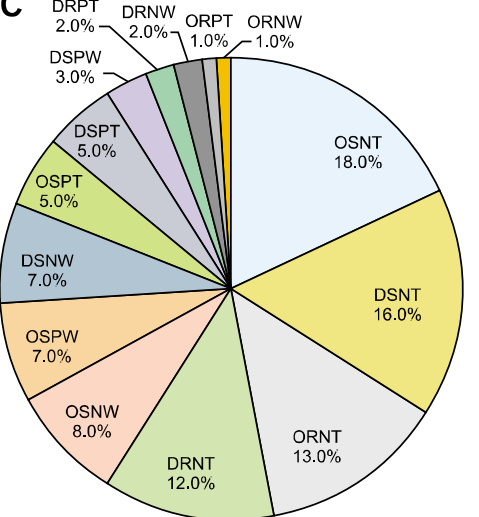

F DRPW DRPT ORPW

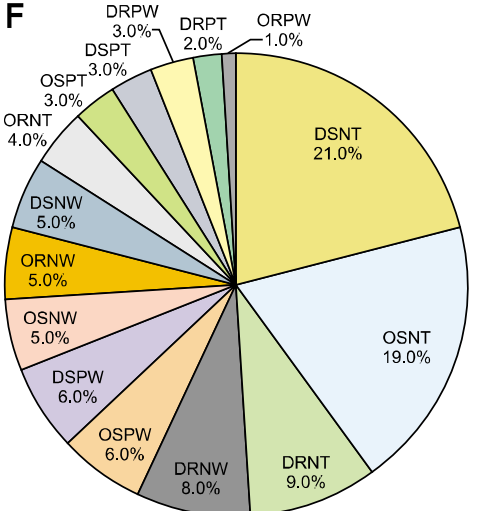

Fig. 2. The distribution of the Baumann skin type according to region. (A) Seoul, (B) Gyeonggi-do/Incheon, (C) Gyeongsangnam-do, (D) Jeolla-do, (E) Gyeongsangbuk-do, (F) Chungcheong-do, (G) Gangwon-do, and (H) Jeju-do. ORNT and DRNT accounted for approximately $40 \%$ in most of the regions, except Jeolla-do. In Jeolla-do, the proportion of DRNT was twice that of the other regions, and that of DSNT was half that of the other regions. O: oily, D: dry, S: sensitive, R: resistant, P: pigmented, N: non-pigmented, W: wrinkled, T: tight.

subjects. The northern and southern parts of South Korea were defined by using a cut-off of $37^{\circ}$ of latitude. In the southern part, the proportion of subjects with sensitive skin was higher than that in the northern part $(34.7 \%$ vs. $28.6 \%)$, a difference that was statistically significant $(p=0.041)$. The distribution of oily or dry, pigmented or non-pigmented, and wrinkled or tight parameters showed no significant differences between the northern and southern parts of South Korea (Fig. 5).

\section{Miscellaneous factors that might have influenced the skin type distribution}

Based on the data collected with the Baumann skin type questionnaire, relationships between skin type distribution 

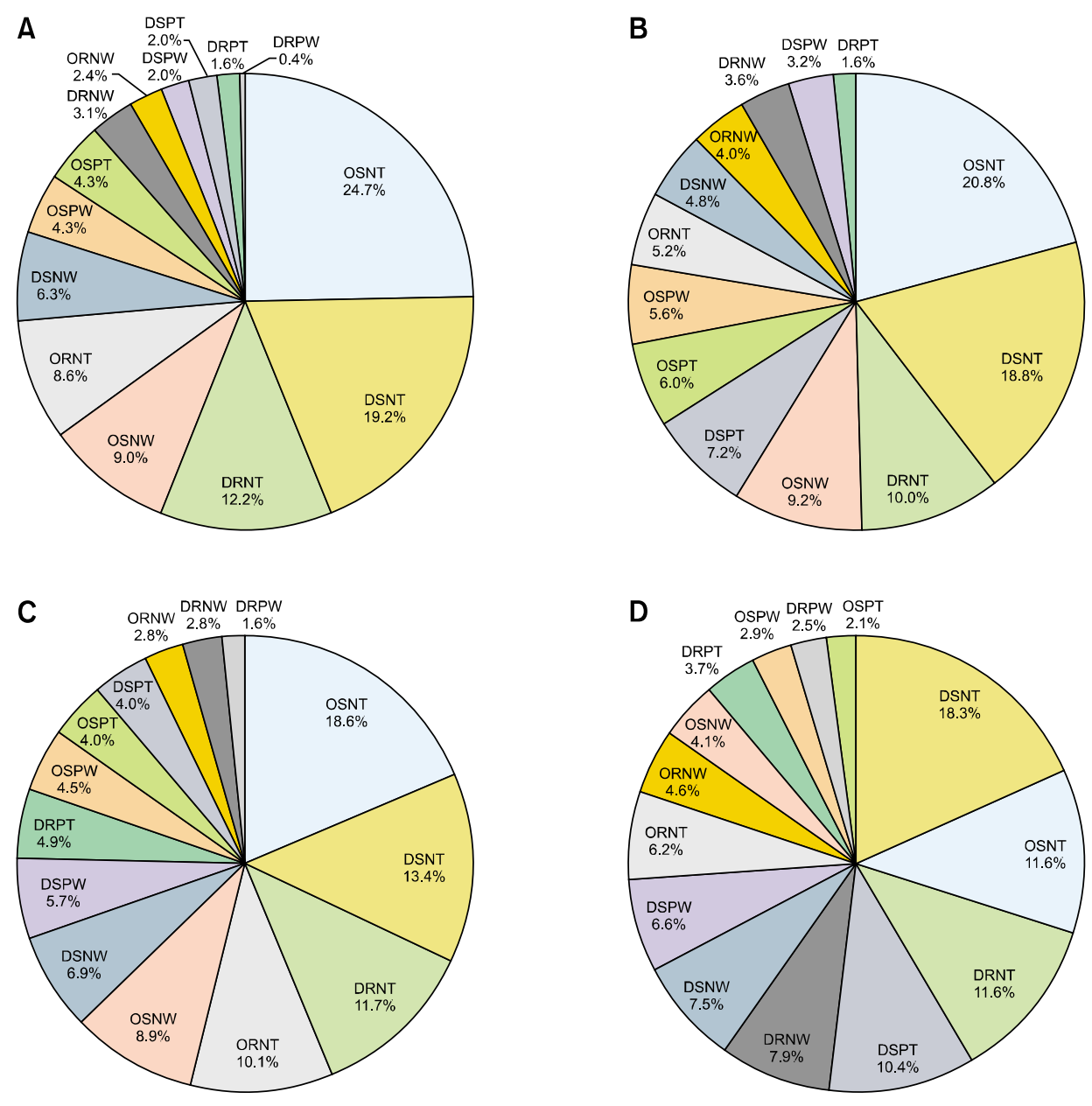

Fig. 3. The distribution of the Baumann skin type according to age. (A) Twenties, (B) thirties, (C) forties, and (D) fifties. The proportion of OSNT showed a decreasing tendency as the age of the survey participants increased. O: oily, D: dry, S: sensitive, R: resistant, P: pigmented, $\mathrm{N}$ : non-pigmented, $\mathrm{W}$ : wrinkled, $\mathrm{T}$ : tight.
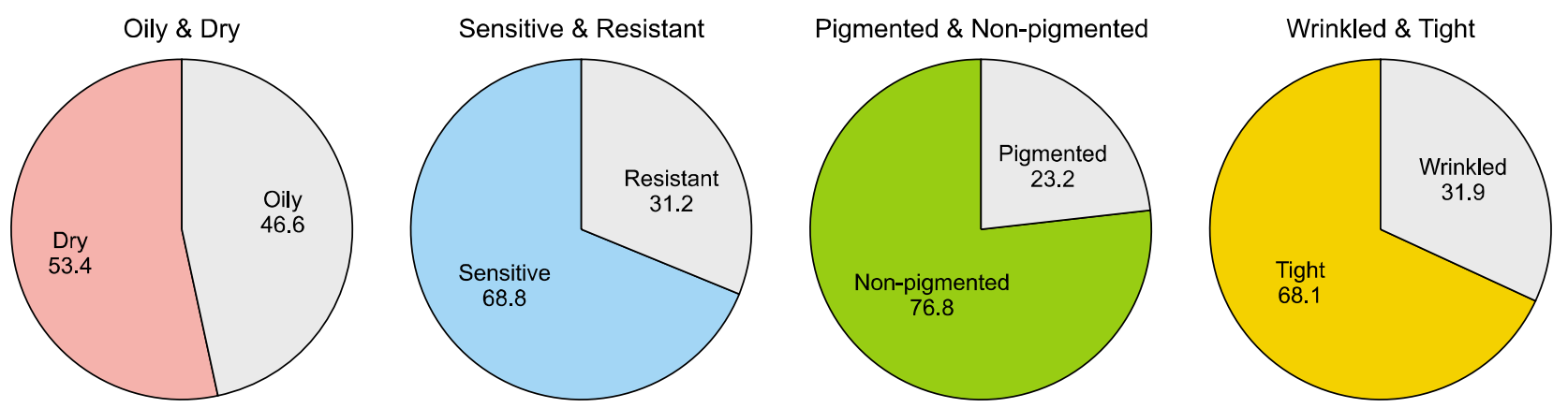

Fig. 4. The distributions of the oily and dry, sensitive and resistant, pigmented and non-pigmented, and wrinkled and tight skin types among Korean women.

and smoking or drinking habits, UV exposure, and blood type were analyzed.

\section{1) Smoking habits}

Information about whether the subject was exposed to smoking was collected. Any subject who was currently smoking or indirectly exposed to a smoking environment (current smoker's wife, child, or a co-worker) was defined as a smoker. Only subjects who were not exposed to any smoking environment were defined as non-smokers. Among the subjects, $18.4 \%$ were direct or indirect smokers (Fig. 6). The proportion of the wrinkled skin type was significantly higher in smokers than in non-smokers $(66.3 \%$ vs. $24.1 \%, p=0.022)$. In addition, the proportion of the subjects with an oily skin type was slightly higher in the smokers $(47.8 \%$ vs. $46.3 \%, p=0.023)$. 

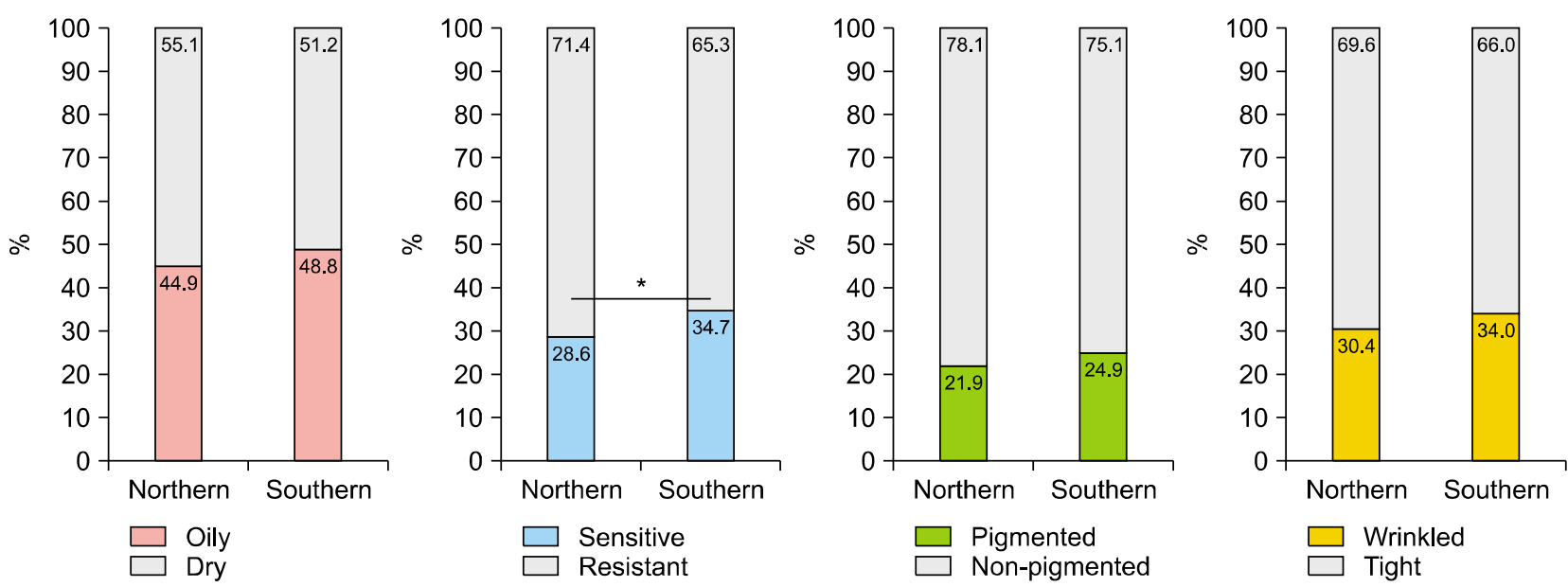

Fig. 5. Comparison of the Baumann skin type in the northern and southern parts of South Korea. The proportion of the sensitive types was higher in the region with a latitude of $<37$ degree $\left({ }^{*} p=0.041\right)$. The proportions of the oily, wrinkled, and pigmented types were higher in the region with a latitude of $<37$ degree but the differences did not reach statistical significance $(p>0.05)$.

\section{2) Blood type, use of sunscreen, occupational UV ray exposure, and drinking habits}

The distribution of Baumann's 4 parameters were analyzed according to the blood type of the subjects, use of sunscreen, drinking habits, and occupational UV exposure. However, no statistically significant differences between groups were observed.

\section{DISCUSSION}

Since Rubinstein introduced the first skin type system in 1910, more personalized skin type systems have emerged ${ }^{2}$. Introduced in 1975, the Fitzpatrick scale is focused on the skin reaction to UV radiation. Therefore, it is highly related to sunburn or pigmentation tendency ${ }^{3}$. The Fitzpatrick scale is so popular in the dermatological community that it is almost considered as a synonym to skin type itself. Among Koreans, according to the study of Youn et al. ${ }^{4}$, the most common Fitzpatrick skin type is type III, and types III, IV, and V account for $88.8 \%$ of the total population. However, the Fitzpatrick skin scale has limitations in that it cannot predict skin reactions to mechanical trauma or laser treatment. Accordingly, a number of skin type systems have been introduced for the purpose of evaluating diverse skin conditions such as pigmentation or treatment response $\mathrm{e}^{5-10}$.

In 2008, Kim et al. ${ }^{11}$ claimed that the wrinkled and the dry skin type were more frequently observed in Koreans who were outdoor workers. However, these previous studies of Koreans had their limitations in that they were based on data from relatively small populations and that they relied only on the Fitzpatrick scale, excluding other skin conditions.
Therefore, a more comprehensive study that can represent the skin characteristics of the Korean population is needed.

The BST system, which was proposed in 2004 by Baumann et al., is being increasingly applied in diverse fields $^{1,12}$. The BST system has 4 distinguished parameters that are highly associated with characteristic features of skin. The dry or oily and sensitive or resistant types are associated with skin barrier function, and the pigmented or non-pigmented, and wrinkled or tight types are related to skin phototype.

In general, the most common skin type systems define a person's skin condition as dry, oily, neutral, or combination type ${ }^{13}$. According to previous studies, the dry and combination types equally accounted for $30 \%$, and the oily and neutral types both accounted for $15 \%$ of the Korean female population ${ }^{14}$. However, considering that various combinations can be present in the forehead, cheeks, and perinasal area, and diverse environmental factors may influence skin condition, a more sophisticated method of diagnosis is necessary. In this study, the oily and dry type accounted for $46.6 \%$ and $53.4 \%$, respectively. The description "sensitive skin" is commonly used by cosmetics companies, but an established definition has yet to be proposed. In general, sensitive skin implies increased reactivity to external stimuli, including cosmetic products $^{15}$. Manifestation of sensitive skin is owing to heightened neurosensory input, enhanced immune responsiveness, and disrupted barrier function. Sensitive skin is also provoked by changes in cutaneous nerve endings, increased neurotransmitters, abnormalities in neurotransmission, or chronic injury of cutaneous nerve endings ${ }^{16}$. Although the diagnosis of sensitive skin is highly sub- 

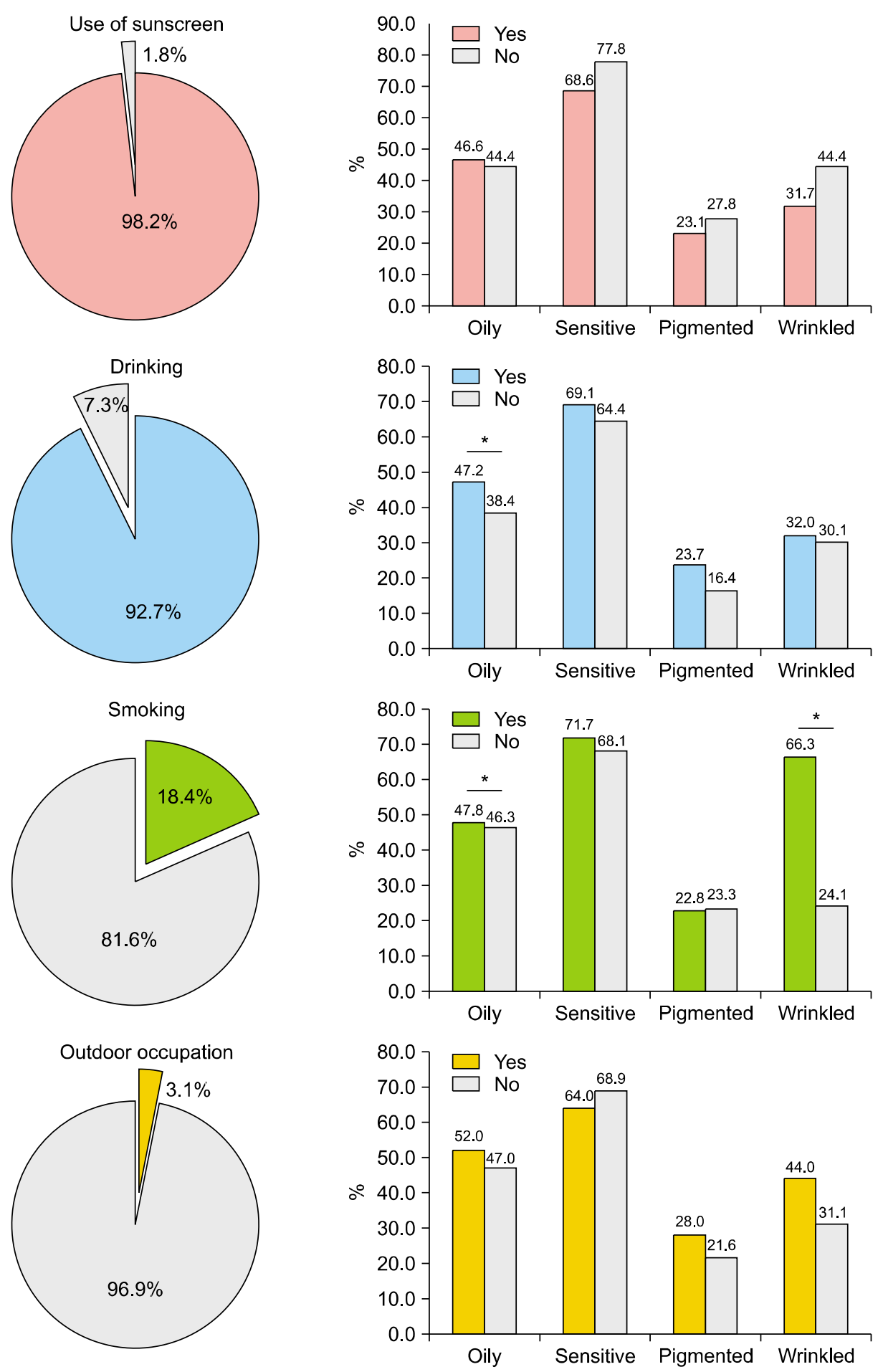

Fig. 6. Distribution of Baumann skin type according to use of sunscreen, drinking, smoking, and professional ultraviolet exposure. The proportion of oily type was significantly higher in drinkers and smokers, and the proportion of wrinkled type was 2.75 times higher in smokers $(* p<0.05)$.

jective, $55.7 \%$ of Korean women and $38.9 \%$ of Korean men had sensitive skin in one epidemiologic study ${ }^{17}$, and the proportion of men with sensitive skin is steadily increasing $^{18}$. Compared with the results of previous studies, the proportion of women with sensitive skin in this study is relatively high $(68.8 \%)$. This result might be presumably be due to the wide range of information including sensi- tivity to cosmetics and history of allergy disease obtained from the BST questionnaire, which results in more comprehensive evaluation of skin conditions that other systems.

In addition, the proportion of women with sensitive skin was the highest in Seoul. From the perspective of environmental factors, these differences in frequencies may be 
due to the degree of urbanization, air pollution, or the location of the city. Although precise causation has not been revealed, various environmental factors might have influenced the differences in the distribution of sensitive skin types.

Skin pigmentation can be evaluated by using the tristimulus colorimeter, narrow-band reflectance spectrophotometer, or DermaSpectrometer (Courage + Khazaka Electronic $\mathrm{GmbH}$, Cologne, Germany). UV irradiation and skin phototype play an important role in determining the pigmented or non-pigmented skin type. Using this parameter, the possibility of skin pigmentation after dermatologic procedures such as laser treatment can be predicted. In previous studies, pigmented skin type was relatively common, comprising $\geq 80 \%{ }^{4,19}$, an approximately 3 -fold higher prevalence that that found in the current study. This is partially due to the difference between the Fitzpatrick scale and the BST system. In the Fitzpatrick scale, skin color is the most important factor in determining skin type. In the BST system, the pigmented skin type is diagnosed based on comprehensive information regarding skin pigmentation tendency after trauma or pharmaceutical challenges, family history of pigmented skin disease, and skin reaction to UV irradiation. Although approximately $80 \%$ of Koreans have dark skin of Fitzpatrick type III or higher, the proportion with the BST pigmented type, which is characterized by vulnerability to pigmentation or pigmentary skin disease, is much lower.

In the BST system, the most distinguishing feature of the wrinkled or tight parameter is that it is influenced by patient-modifiable factors such as UV protection, drinking, and smoking. According to data from the Korea Centers for Disease Control and Prevention, the female smoking rate in South Korea has decreased since $2012(7.9 \%)$, reaching $5.7 \%$ in 2014 . In this study, the wrinkled and tight types accounted for $31.9 \%$ and $68.1 \%$ of the subjects, respectively. Furthermore, the proportion with the wrinkled type was significantly higher in the smoking group $(p<0.05)$.

To broadly reflect the life patterns and medical history of subjects when analyzing the distribution of Baumann's skin type, we collected not only the age of the subjects but also subjects' information such as sunscreen use, professional UV exposure, and drinking and smoking habits. $\mathrm{ABO}$ blood type was studied as part of the medical history. Despite the effort to investigate the relationship between this information and the distribution of BST, no significant correlation was observed except for a few cases such as use of alcohol and smoking and the distribution of oily skin, and smoking and the distribution of wrinkled skin. This might be because in this study, factors such as the use of sunscreen or professional UV exposure were only qualitatively analyzed by a yes or no question, and the groups with and without sunscreen, and the groups with and without occupational UV exposure showed a severe imbalance in their numbers. Further studies that will overcome these limitations are necessary.

The distribution of Baumann skin type except for the sensitive type showed no significant difference between the southern and northern part of South Korea. In March, the UV indices in the northern and southern parts of South Korea are 5.0 and 7.0 , respectively, which means that the probability of skin sun damage is 1.4 times higher in southern South Korea than in the northern part ${ }^{20}$. Considering that the correlation between UV exposure and the occurrence of sensitive skin types is limited, and that in this study there were no significant correlations between UV exposure and more closely related indicators such as wrinkled or pigmented skin, the effect of latitude on the distribution of skin type needs further investigation.

This study has its limitations in that subjective interpretation could cause bias, especially in parts 3 and 4 of the questionnaire. However, the concept of skin type itself could be considered a matter of subjective interpretation, and more importantly, each part of the BST questionnaire consists of complementary questions that can provide a comprehensive interpretation of the subject's skin type. For example, the skin pigmentation tendency is assessed by objective criteria, such as personal or family history of pigmentary disease and the exact number of nevus on the sun-exposed areas. The result of part 4 is also calculated from objective criteria, such as the frequency of UV exposure, smoking, and consumption of vegetables and fruits. Furthermore, given that the study was targeting 1,000 Korean women assigned proportionally according to the population of each province of Korea, it has many advantages over other studies.

In this study, the most common skin type in the Korean female population was OSNT. As the age of the subjects increased, the proportions of the pigmented and wrinkled types also increased, whereas that of the oily type decreased significantly. The proportion of sensitive skin was highest $(75.1 \%)$ in the thirties age group. The wrinkled type was significantly more common among smokers.

The BST system can draw a comprehensive diagnosis of skin conditions based on evaluations of moisturization and sebum production, reactivity to environmental irritations, skin pigmentation after UV irradiation, and skin tightness. From this, a more personalized approach to diverse skin conditions can be implemented. Further evaluation of the mechanisms that link the related factors identified in this study is necessary. 


\section{ACKNOWLEDGMENT}

This article was supported by Byeong-Deog Park, Neopharm USA.

\section{CONFLICTS OF INTEREST}

The authors have nothing to disclose.

\section{REFERENCES}

1. Baumann L. The skin type solution: a revolutionary guide to your best skin ever. New York: Bantam Books, 2006:4-26.

2. Roberts WE. Skin type classification systems old and new. Dermatol Clin 2009;27:529-533, viii.

3. Fitzpatrick TB. The validity and practicality of sun-reactive skin types I through VI. Arch Dermatol 1988;124:869-871.

4. Youn JI, Choe YB, Park SB, Suh DH, Park YK, Ahn SK, et al. The Fitzpatrick skin type in Korean people. Korean J Dermatol 2000;38:920-927.

5. Kawada A. UVB-induced erythema, delayed tanning, and UVA-induced immediate tanning in Japanese skin. Photodermatol 1986;3:327-333.

6. Glogau RG, Matarasso SL. Chemical peels. Trichloroacetic acid and phenol. Dermatol Clin 1995;13:263-276.

7. Lancer HA. Lancer Ethnicity Scale (LES). Lasers Surg Med 1998;22:9.

8. Fanous N. A new patient classification for laser resurfacing and peels: predicting responses, risks, and results. Aesthetic Plast Surg 2002;26:99-104.

9. Goldman MP. Universal classification of skin type. In:
Shiffman MA, Mirrafati SJ, Lam SM, Cueteaux CG, editors. Simplified facial rejuvenation. Berlin: Springer Berlin Heidelberg, 2008:47-50.

10. Taylor S, Westerhof W, Im S, Lim J. Noninvasive techniques for the evaluation of skin color. J Am Acad Dermatol 2006;54(5 Suppl 2):S282-S290.

11. Kim YS, Park KS, Kang SK. Relationship between occupational quality and skin type through Dr. Baumann's skin analysis. J Kor Soc Cosm 2008; 14:589-594.

12. Baumann L. Cosmeceuticals and cosmetic ingredients. New York: McGraw-Hill Education, 2015:1-18.

13. Youn SW, Park KC. Measurements of facial sebum secretion and facial skin type considering sebum secretion. J Cosmet Dermatol 2006;3:5-10.

14. Kim CS, Chun IK, Kim YP. A study of skin surface lipids analysis of the Koreans. Korean J Dermatol 1992;30:1-7.

15. Lee SH, Lee SE, Ahn SK, Hong SP, Kim HJ, Lee HK, et al. Skin barrier. Seoul: Ryo Moon Gak, 2010:279-290.

16. Draelos ZD. Sensitive skin: perceptions, evaluation, and treatment. Am J Contact Dermat 1997;8:67-78.

17. Ryu HS, Kim DW, Lee SJ, Na GY, Chung SL. Statistical observation of sensitive skin and evaluation of subjective irritation using lactic acid sting test. Korean J Dermatol 2002;40:874-885.

18. Farage MA. How do perceptions of sensitive skin differ at different anatomical sites? An epidemiological study. Clin Exp Dermatol 2009;34:e521-e530.

19. Chung JH, Koh WS, Youn JI. Relevance of skin phototyping to a Korean population. Clin Exp Dermatol 1994; 19:476-478.

20. Fioletov V, Kerr JB, Fergusson A. The UV index: definition, distribution and factors affecting it. Can J Public Health 2010;101:I5-I9. 
Appendix 1. Baumann's skin type questionnaire.

Part One (Oily vs. Dry)

1. After washing your face, don't apply any moisturizer, sunscreen, toner, powder, or other products. Two to three hours later, look in a mirror under bright lights. Your forehead and cheeks feel or appear:
A. Very rough, flaky, or ashy
B. Tight
C. Well hydrated with no reflection of light
D. Shiny with reflection of bright light

11. Two to three hours after applying moisturizer your cheeks are:
A. Very rough, flaky, or ashy
B. Smooth
C. Slightly shiny
D. Shiny and slick, or I do not use moisturizer

Part Two (Sensitive vs. Resistant)

1. You get red bumps on your face:
A. Never
B. Rarely
C. At least once a month
D. At least once a week

18. You get redness, itching, or swelling from make-up, sunscreen, or skin care products:
A. Never
B. Sometimes
C. Frequently
D. Always
E. I do not use these products. (Note: answer D if you don't use them because of redness, itching, or swelling)

Part Three (Pigmented vs. Non-pigmented)

1. After you have a pimple or ingrown hair, it's followed by a dark brownish/black spot:
A. Never
B. Sometimes
C. Frequently
D. Always
E. I never get pimples or ingrown hairs.

14. Do you have a history of melanoma yourself or in your immediate family:
A. No
B. One person in my family
C. More than one person in my family
D. I have a history of melanoma
E. Unsure

Part Four (Wrinkled vs. Tight)

1. Do you have facial wrinkles?
A. No, not even with movement such as smiling, frowning, or lifting my eyebrows
B. Only when I move, such as smiling, frowning, or lifting my eyebrows
C. Yes, with movement and a few at rest without movement
D. Wrinkles are present even if I'm not smiling, frowning, or lifting my brows. 
21. What's in your ethnicity? (Please choose best answer.)
A. African-American/Caribbean/Black
B. Asian/Indian/Mediterranean/Other
C. Latin-American/Hispanic
D. Caucasian

The questionnaire consists of a total of 63 questions, which is to lengthy to present in full in this manuscript. Furthermore, according to the contract with the publisher, presentation of the whole questionnaire as supplemental data is prohibited. Therefore the authors respectfully request that readers interested in the entire questionnaire refer to reference \#1.

Appendix 2. The proportions of the subjects with oily, sensitive, pigmented, and wrinkled types according to age group. Younger subjects showed a higher proportion of oily skin $(p<0.05$ between each age group), whereas older subjects were more prone to having pigmented and wrinkled skin $(p<0.05$, between each age group). Sensitive skin type was the most common in subjects in their thirties $(p<0.05$, between each age group).

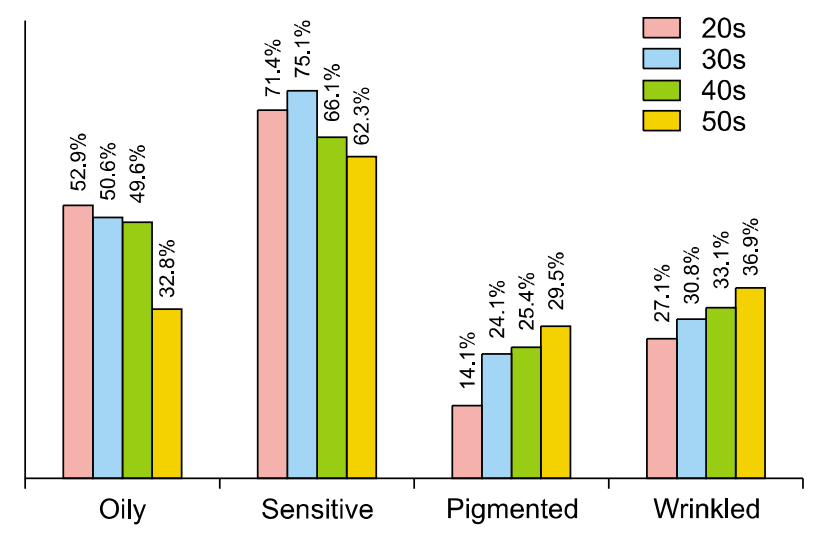

Appendix 3. The proportions of the subjects with the oily, sensitive, pigmented, and wrinkled types according to province. The distributions of the oily, pigmented, and wrinkled types did not show significant differences according to region. The proportion of sensitive skin was highest in Seoul and lowest in Gyeongsangnam-do. The differences of prevalence of sensitive skin type according to province were statistically significant between each age group $(p<0.05)$.

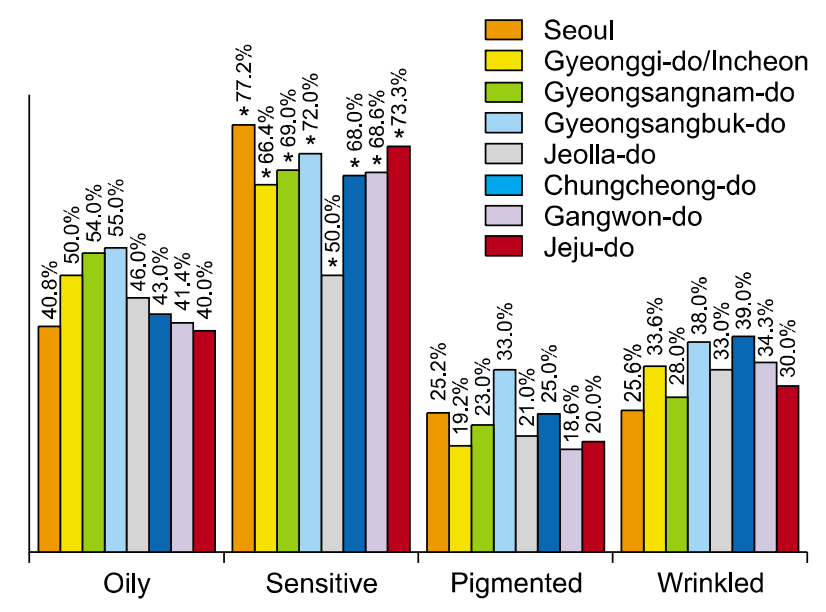

